\title{
Muséologies
}

Les cahiers d'études supérieures

\section{Les musées de l'école et de l'éducation : un champ muséal quantitativement significatif mais difficile à cerner}

\section{Myriam Boyer}

Volume 5, numéro 2, printemps 2011

URI : https://id.erudit.org/iderudit/1033517ar

DOI : https://doi.org/10.7202/1033517ar

Aller au sommaire du numéro

Éditeur(s)

Association Québécoise de Promotion des Recherches Étudiantes en Muséologie (AQPREM)

ISSN

1718-5181 (imprimé)

1929-7815 (numérique)

Découvrir la revue

Citer cet article

Boyer, M. (2011). Les musées de l'école et de l'éducation : un champ muséal quantitativement significatif mais difficile à cerner. Muséologies, 5(2), 104-129. https://doi.org/10.7202/1033517ar
Résumé de l'article

En étudiant la gestion, les politiques d'acquisition, de documentation et de valorisation d'un bon nombre d'institutions muséales européennes vouées à l'école et à l'éducation, Myriam Boyer établit une réflexion sur la pérennisation de leurs collections et sur les enjeux liés à leur diffusion. Fondé sur une étude de terrain rigoureuse menée entre 2005 et 2007, le panorama quantitatif et qualitatif qu'elle a dressé lui permet de poser une réflexion démontrant qu'une corrélation peut être établie entre les missions de ces musées et l'esprit du temps d'une société. Pour l'auteure, la sauvegarde de ce patrimoine repose sur deux conditions : la reconnaissance du rôle patrimonial et social de ces collections et le ralliement et la diversification des publics. 
Myriam Boyer

Les musées de l'école et de l'éducation : un champ muséal quantitativement significatif mais difficile à cerner

En étudiant la gestion, les politiques d'acquisition, de documentation et de valorisation d'un bon nombre d'institutions muséales européennes vouées à l'école et à l'éducation, Myriam Boyer établit une réflexion sur la pérennisation de leurs collections et sur les enjeux liés à leur diffusion. Fondé sur une étude de terrain rigoureuse menée entre 2005 et 2007 , le panorama quantitatif et qualitatif qu'elle a dressé lui permet de poser une réflexion démontrant qu'une corrélation peut être établie entre les missions de ces musées et l'esprit du temps d'une société. Pour l'auteure, la sauvegarde de ce patrimoine repose sur deux conditions: la reconnaissance du rôle patrimonial et social de ces collections et le ralliement et la diversification des publics.

Myriam Boyer est agrégée d'histoire, diplômée de l'École du Louvre, docteure en histoire des techniques - muséologie du Conservatoire national des arts et métiers. De 2004 à 2010, elle a été chargée de conservation et de recherche au Musée national de l'éducation de Rouen (France), responsable du pôle conservation, chargée du projet de chantier des collections et du transfert de ces dernières dans de nouveaux locaux. Depuis 2009, elle est codirectrice du groupement européen d'intérêt économique "Preservation of the School History Heritage in Europe EEIC/Bewahrung der materiellen Schulkultur in Europa EWIV", dont l'objectif est de promouvoir les musées de l'école et de l'éducation. myriam.boyer@cned.fr 


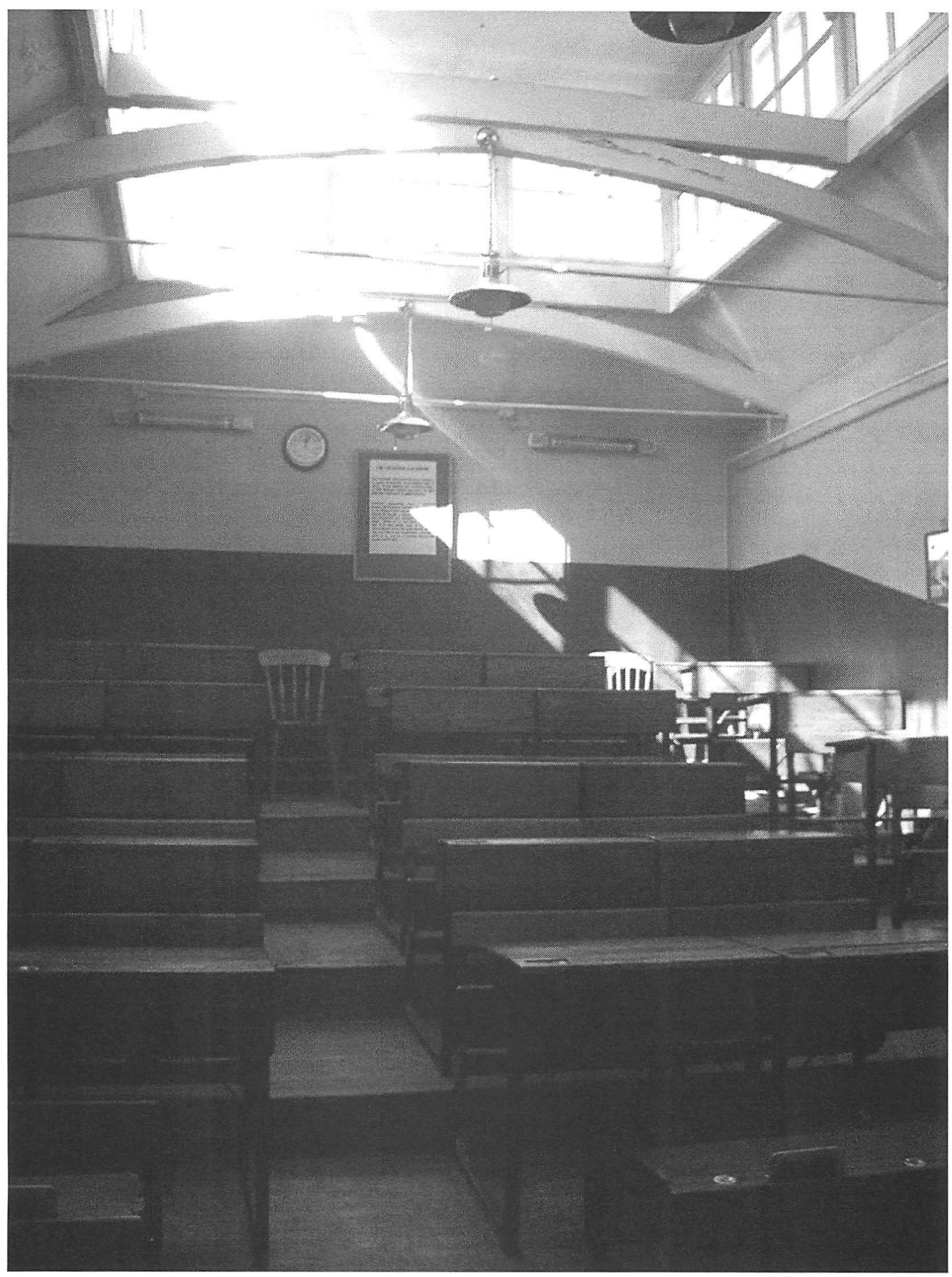

"The Galleried classroom» (1853)

British Schools Museum Hitchin.

Photographie: Myriam Boyer, 2007 
Une enquête menée en 1998-1999 par le Schulmuseum de BergischGladbach en Allemagne souhaitait dresser un panorama des musées de l'école: le questionnaire, envoyéà 100 musées de l'école et collections historiques sur l'école, ne reçut que 15 réponses de musées allemands et étrangers. (KOLLOCH, Ursula. "Das Schulmuseum. Was soll, kann und darf es leisten? " [Le musée de l'école. Quelle est sa fonction?]. Dokumentation. International Symposium for School Museums and School History Collections / Internationales Symposium für Schulmuseen und Schulgeschichtliche Sammlungen. Friedrichshafen/ Weingarten: Schulmuseum Friedrichshafen / Forschungstelle für Schulgeschichte, Pädagogische Hochschule Weingarten, 2000 , p. 38-47.)

Plus récemment, une étude comparative sur les musées de l'éducation d'Europe de l'Ouest et d'Europe de l'Est a porté sur 26 d'entre eux. (RIBARIC, Mateja. "Basic Information about Eastern European Schoolmuseums and Comparison with Those in Western Europe ". Zur Rolle von Schul - und Kindermuseum im 21. Jahrhundert [Le rôle des musées de l'école et de l'enfance au XXIe siècle]. Berlin: LITVerlag, 2008, p. 67-81.)

2

A titre d'exemple, trois sites Internet proposaient des listes de musées de l'école, de l'éducation ou de collections de patrimoine scolaire.

A l'échelle mondiale, l'Université de Louvain en Belgique a recensé, en octobre 2002, 490 sites de 449 musées et collections traitant de l'histoire de l'éducation et de la petite enfance.

<Schulmuseum.net>, créé en mars 2004, est un portail allemand des musées de l'école, des collections historiques sur l'école et des musées conservant du matériel scolaire en Europe. II liste 245 institutions et est particulièrement fourni pour l'Allemagne (163 musées mentionnés en mars 2005), l'Autriche ( 32 musées répertoriés en mars 2006) et la Suisse (17 musées en juillet 2003), mais lacunaire concernant "les autres pays".

A l'échelle mondiale, le panorama des musées de l'école et de l'éducation s'apparente à une immense mosaïque composée d'institutions muséales d'une densité variable selon les régions. Diverses tentatives ont été menées depuis les années 1990 pour identifier' et recenser ${ }^{2}$ les musées de l'école, de l'éducation et les collections de patrimoine scolaire. Aucune pourtant n'est parvenue à cerner ce champ protéiforme de manière exhaustive. La recension la plus précise à ce jour reste la vaste étude menée par l'équipe scientifique du Museo Pedagóxico de Galicia (MUPECA) et des chercheurs de l'Université de Saint-Jacques de Compostelle (Espagne), de 1999 à novembre $2003^{3}$. Elle identifie 683 musées répartis dans 42 pays appartenant prioritairement aux continents européen et américain. L'Europe, avec ses 442 institutions recensées, concentre à elle seule près de $65 \%$ du corpus. Les pays les plus pourvus en musées sont, selon le repérage, l'Allemagne (103), la Suède (64), la France (46), suivis de l'Autriche (38), de l'Espagne (36), de la Norvège (33), de la Grande-Bretagne (28) et de la Suisse (15). Le continent américain (192 institutions) abrite $28 \%$ des institutions et les États-Unis (141) et le Canada (36) en concentrent l'essentiel. Les autres continents sont moins dotés. En leur sein, dominent les pays les plus occidentalisés. En Océanie $(5,8 \%)$, seules l'Australie (38) et la Nouvelle-Zélande (2) comptent des musées de l'éducation. L'Asie est moins riche encore $(1,1 \%)$ : la première place revient au Japon (6), devant Dubaï (1) et la Malaisie (1). Un seul musée a été recensé en Afrique.

Les chiffres avancés dans cette étude sont très nettement sous-évalués et la réalité certainement déformée en raison de la méthode choisie et de la définition du corpus 4 .

A l'échelle nationale, le site <UKschoolmuseums.org.uk> présente les quinze musées de l'école qui, au Royaume-Uni, se réunissent en conférence annuelle depuis 1995. 3

PEÑA SAAVEDRA, Vicente (dir.) et Manuel FERNÁNDEZ GONZÁLEZ et Oscar MONTERO FEIJOO. Os museos da educación en Internet [Les musées de l'éducation sur Internet]. SaintJacques de Compostelle: Xunta de
Galicia, Consellería de Educación e Ordenación Universitaria, Museo Pedagóxico de Galicia, 2004.

4

La recension des musées de l'école et de l'éducation français menée par le Musée national de l'éducation (Rouen) a, par exemple, permis de recenser en 2006-2007 108 institutions muséales (et non 46 comme dans la recension du MUPECA). 
En effet, le parti pris a consisté à repérer les musées pédagogiques ainsi que les musées de l'éducation et de l'école présents sur Internet, c'est-à-dire qui soit disposent de leur propre site, soit sont hébergés sur un autre site. De plus, une double restriction a été imposée au corpus. Seules les institutions muséales répondant à la définition internationale proposée par le Conseil international des musées (ICOM), conservant et exposant des objets et matériaux relevant du champ de l'éducation, ont été retenues. Les expositions ou musées virtuels que l'on voit naître sur le web ont été par contre inclus. Par ailleurs, les musées scientifiques, les musées des jeux et jouets ainsi que les musées de l'enfance n'ont volontairement pas été recensés dans cette étude car, selon les auteurs, ils n'entretiennent pas de rapport direct avec le domaine de l'enseignement.

En dépit de cette restriction du champ d'investigation, les chiffres avancés permettent de prendre la mesure du poids relatif des musées de l'école et de l'éducation dans les paysages muséaux international et national dont ils ne constituent qu'un infime sous-ensemble.

\section{Un champ muséal marqué par la diversité}

Portés par un fondateur ou une équipe, expressions d'une école pédagogique, produits d'une volonté politique locale ou nationale, lieux de conservation, d'exposition et de diffusion, les musées de l'école et de l'éducation se révèlent extrêmement hétérogènes ne serait-ce que par leur histoire, par leurs collections, par leur statut juridique et administratif, par leurs moyens humains et matériels, par leurs missions et projets et par le dynamisme de leur offre culturelle. La même diversité transparaît quant au lieu qui les abrite, du plus institué, installé dans un vaste bâtiment à l'architecture remarquable et ouvrant ses portes sur les grandes artères d'une capitale, au plus discret, occupant un modeste local dans un établissement scolaire5. Au gré des projets, des moyens et des espaces disponibles, le musée se résume en effet à quelques objets présentés ponctuellement à des visiteurs, formant ou non une salle entière. Très souvent, il propose une salle de classe reconstituée dans
5

Cette diversité était au cœur de la thématique du $\eta^{\text {th }}$ International Symposium for Museums of School Life and School History Collections / $m$. Internationales Symposium für Schulmuseen und Schulgeschichtliche Sammlungen (Kartause Ittingen, Suisse, 21-24 juillet 2005). Les actes de ce symposium ont été partiellement publiés: Zur Rolle von Schul- und Kindermuseum im 27. Jahrhundert. Berlin: LITVerlag, 2008. 


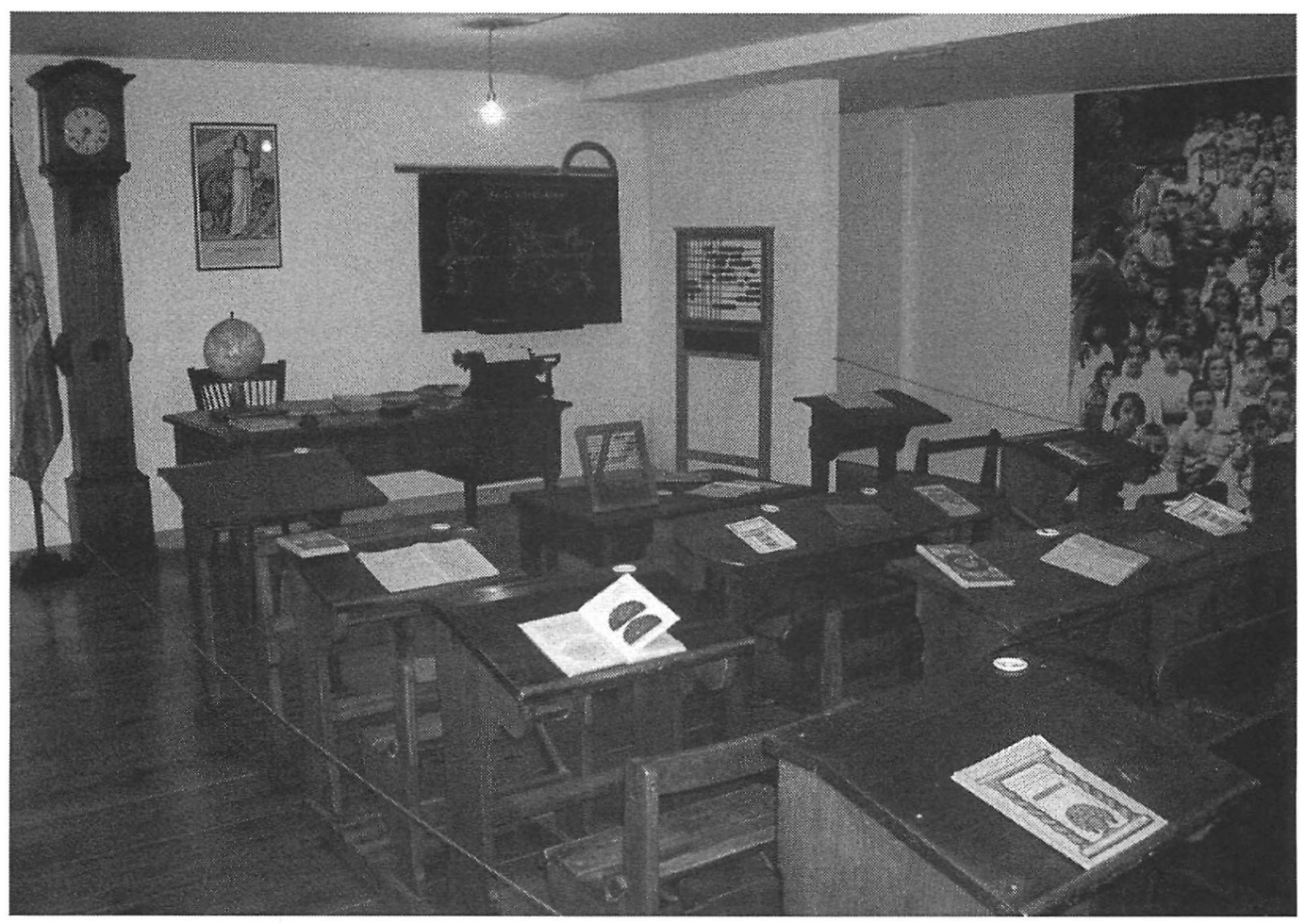

"Aula de Republica 1931-1936"

Museo Pedagóxico de Calicia.

Photographie: Myriam Boyer, 2007 


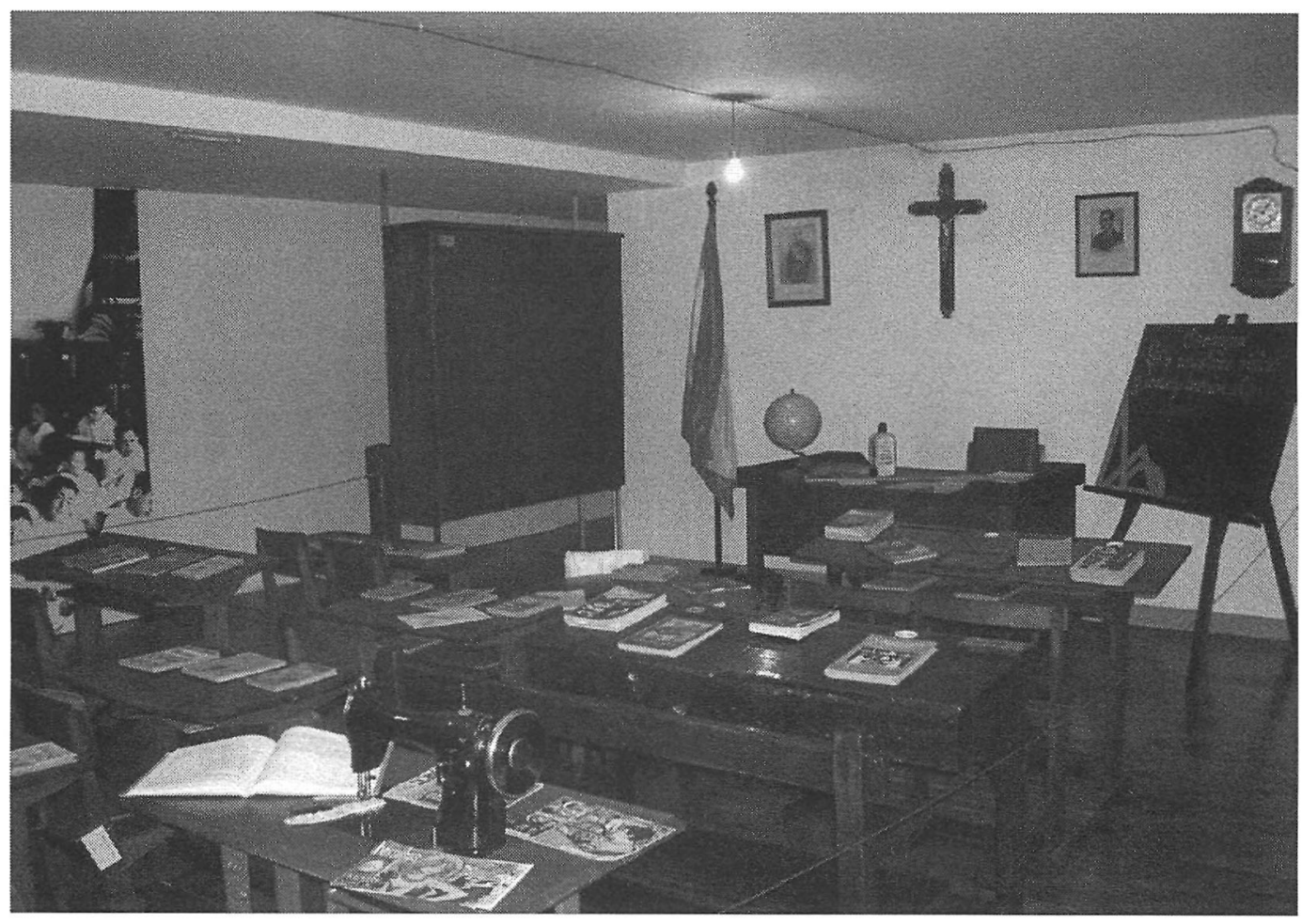

"Aula de Franquismo 1939-1975",

Museo Pedagóxico de Galicia.

Photographie: Myriam Boyer, 2007 


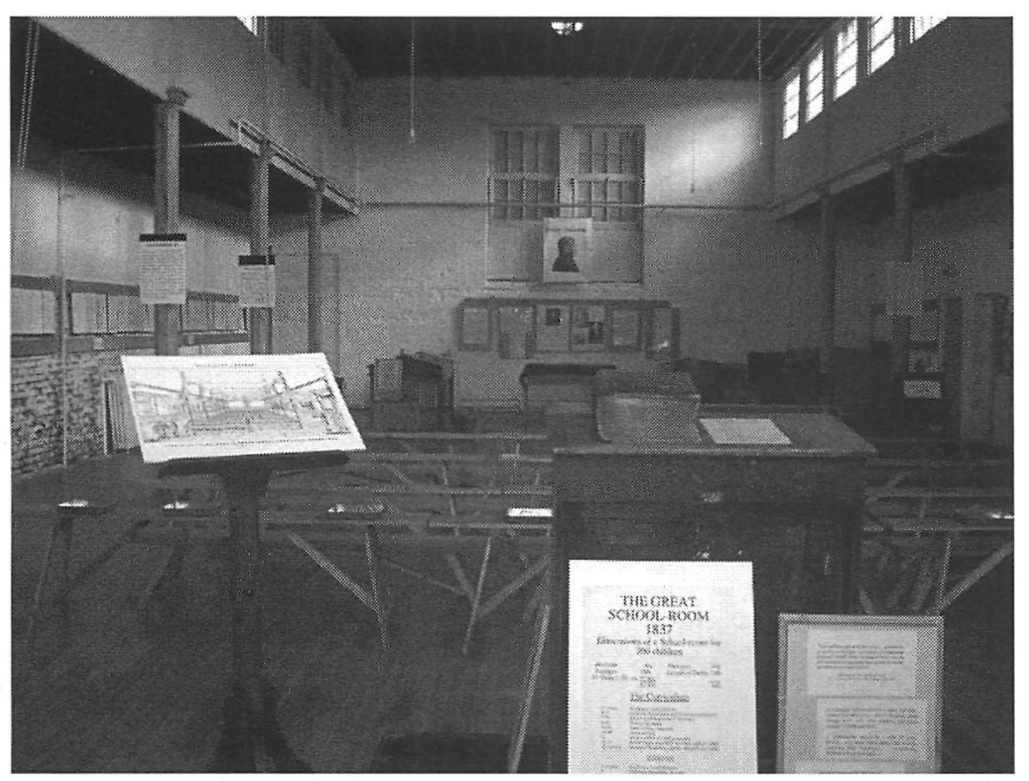

"The great Lancasterian Schoolroom" (1837) British Schools Museum Hitchin. Photographie: Myriam Boyer, 2007

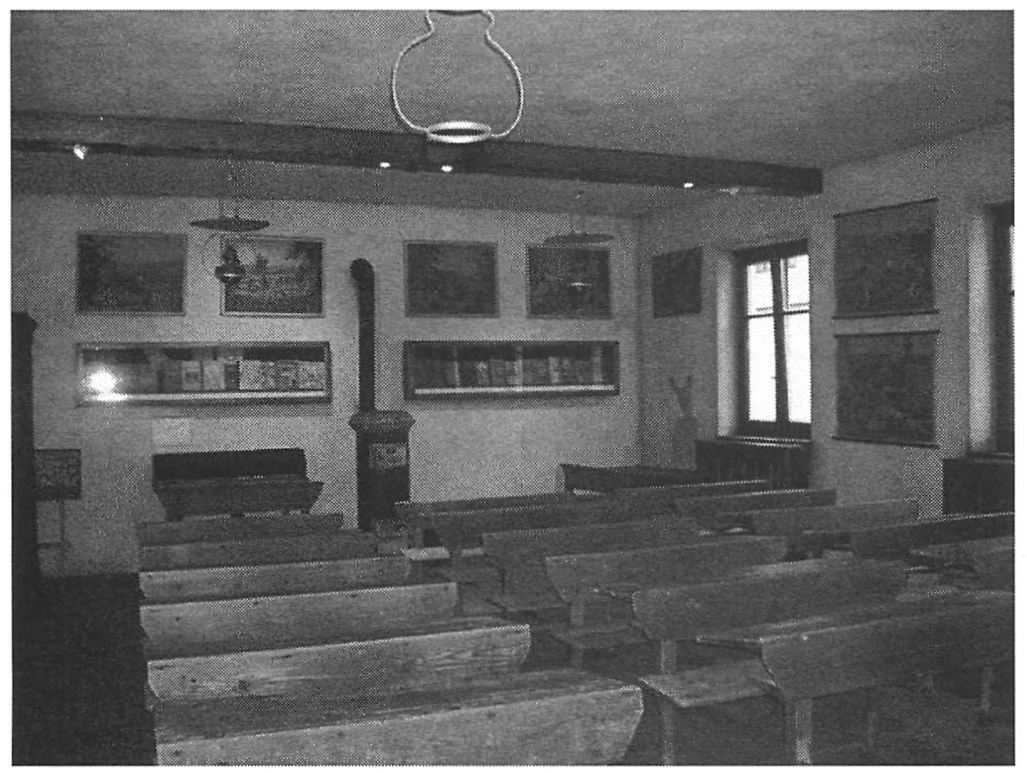

"189o. Deutschklasse : schreiben und lesen". Michelstettner Schule - Niederösterreichisches Schulmuseum, Michelstetten. Photographie: Myriam Boyer, 2007 


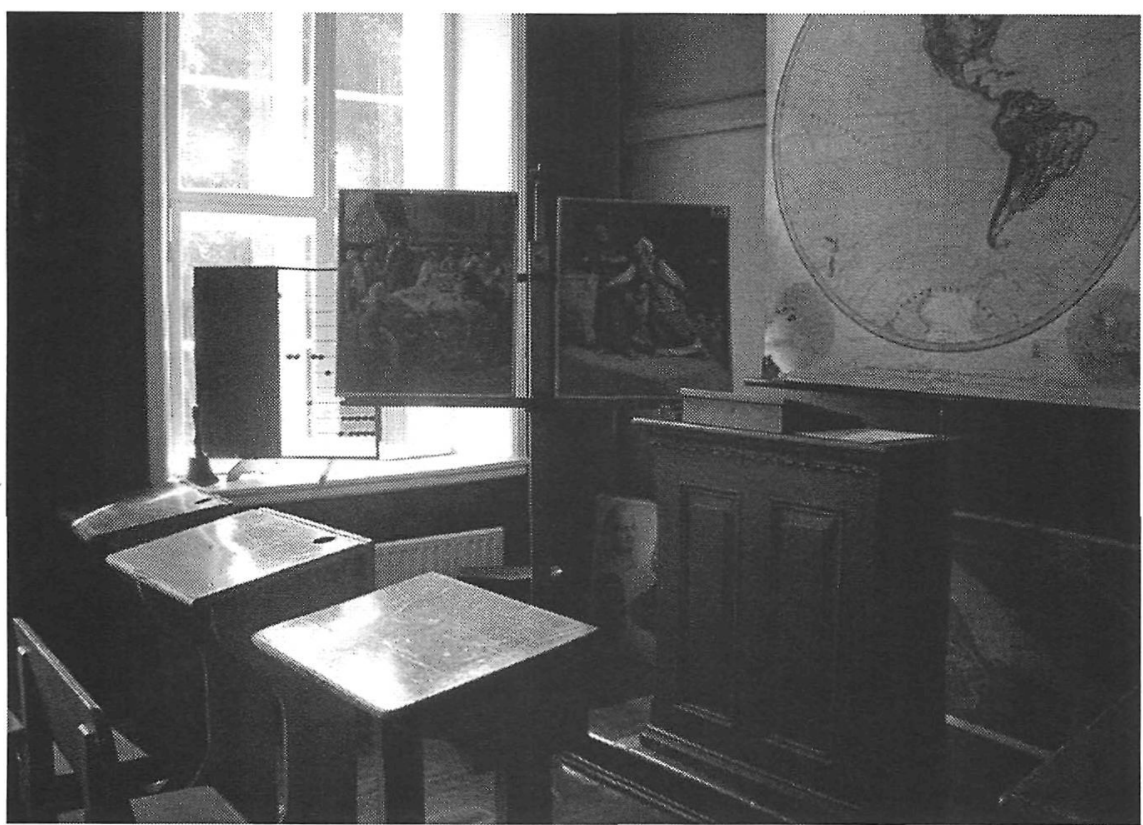

Reconstitution d'une salle de classe de l'école primaire (première moitié du XXe siècle) «Folkeskolens klasserom ", Bergen Skolemuseum. Photographie: Myriam Boyer, 2007 


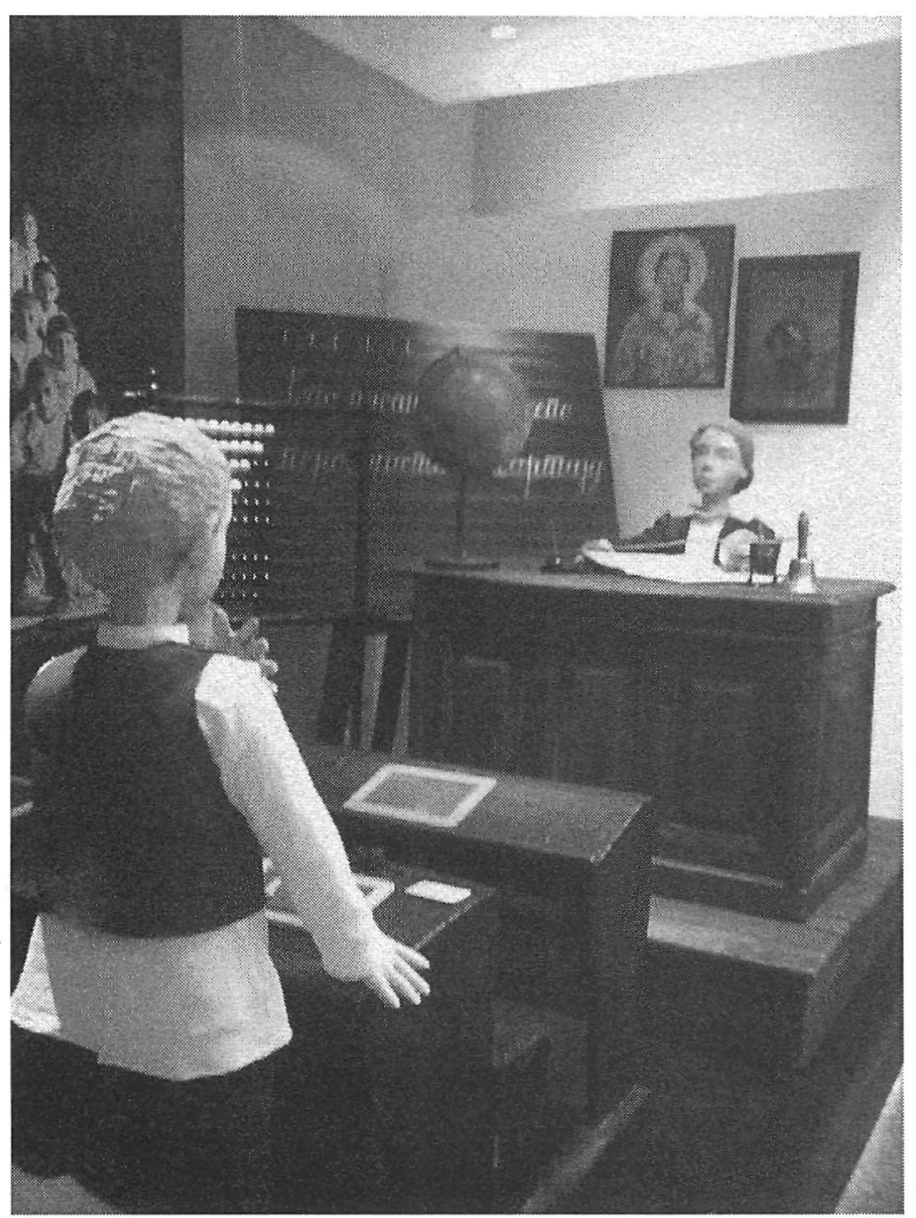

Évocation d'une salle de classe d'une école primaire (vers 1900). Pedagoški muzej de Belgrade.

Photographie : Myriam Boyer, 2007 


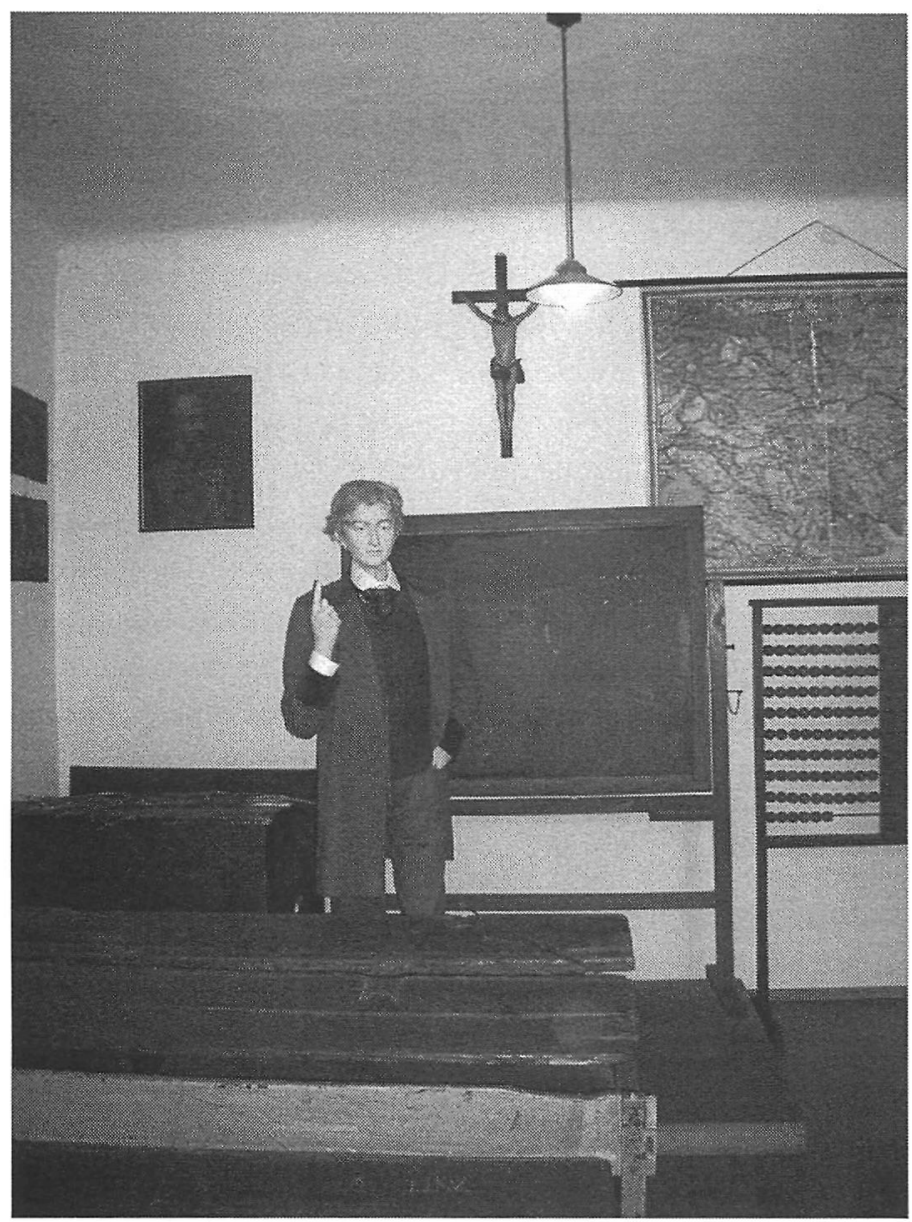

Reconstitution d'une salle

de classe de l'école primaire (vers 1900) Slovenski Šolski Muzej de Ljubljana. Photographie:

Myriam Boyer, 2007 


\section{6}

La définition des fonctions muséales fait l'objet de débats. Nous avons repris celles mentionnées dans la définition du musée adoptée par I'ICOM en 2007 (statuts de l'ICOM adoptés lors de la $21^{\mathrm{C}}$ Conférence générale à Vienne): "Un musée est une institution permanente sans but lucratif au service de la société et de son développement ouverte au public, qui acquiert, conserve, étudie, expose et transmet le patrimoine matériel et immatériel de l'humanité et de son environnement à des fins d'études, d'éducation et de délectation. "Quạtre fonctions principales peuvent être retenues: la conservation (comprenant l'acquisition), l'exposition, la recherche et l'animation (comprenant au sens large toutes les actions en faveur de la diffusion auprès des publics).

7

Le rayonnement du musée a été évalué en fonction de sa fréquentation, de ses publications, des recherches menées, de ses activités, de sa participation aux échanges internationaux et de l'existence d'un site Internet.

\section{8}

Les musées retenus dans le corpus sont les suivants (classement par pays): Allemagne (7): Schulgeschichtliche Sammlung Bremen, Brême; Hamburger Schulmuseum, Hambourg: Sammlung Kindheit und Jugend, Berlin; Schulmusem Nordwürttemberg, Kornwestheim Schulmuseum Friedrichshafen am Bodensee, Friedrichshafen: Schulmuseum - Werkstatt für Schulgeschichte Leipzig, Leipzig: Bayerisches Schulmuseum Ichenhausen, Ichenhausen. Autriche (1): Michelstettner Schule - Niederösterreichisches Schulmuseum, Michelstetten. Belgique (1): Stedelijk Onderwijsmuseum leper, Ypres. Croatie (1): Hrvatski Školski Muzej, Zagreb. Danemark (1): Dansk Skolemuseum, Copenhague. Espagne (1): Museo Pedagóxico de Galicia (MUPEGA), Saint-Jacques

une école rurale qu'il a suffi parfois de maintenir en l'état, après que celle-ci fut fermée comme suite à l'exode rural. D'autres fois, à côté de cette salle unique existent des annexes qui proposent des présențations permanentes ou temporaires et des espaces de stockage qui permettent de conserver le matériel collecté. Quelquefois, un lieu de documentation a été aménagé. Le patrimoine éducatif peut, dans d'autres cas encore, être conservé et présenté dans des institutions muséales - ou non - qui ne lui sont pas exclusivement dédiées. L'étude du MUPECA distingue ainsi parmi les institutions muséales non spécialisées dans l'éducation, des musées de plein air, des musées universitaires, des musées de pays et des centres de patrimoine local, des musées des arts et traditions populaires, des musées ethnographiques et des écomusées, mais aussi des musées de l'enfance et de la jeunesse. Parmi les institutions non muséales se trouvent les établissements scolaires en activité et les centres d'études, de recherche, d'archives et de documentation. Enfin, le patrimoine éducatif peut même n'avoir qu'un caractère virtuel. Une étude comparative est-elle dès lors possible? C'est le pari que nous avons fait en retenant 29 musées situés dans 20 pays européens en fonction de critères objectifs (un contenu thématique centré sur l'école et l'éducation, des collections quantitativement significatives, l'exercice des fonctions muséales classiques que sont la conservation, l'acquisition, la communication, l'exposition et la recherche ${ }^{6}$, des moyens humains et matériels minimums pour exercer ces missions, une certaine notoriété)7 et, reconnaissons-le, d'opportunité et de faisabilité ${ }^{8}$.

de Compostelle. Estonie (1): Eesti Pedagoogika Arhiivmuuseum, Tallinn. Finlande (1): Koulumuseo - Helsingin kaupunginmuseo, Helsinki. France (1): Musée national de l'éducation - INRP Rouen, Rouen. Grande-Bretagne (3): British Schools Museum, Hitchin; Ragged School Museum, Londres; Museum of Reading, Reading. Irlande ( 1 ): Plunkett Museum of Irish Education Dublin. Italie (2): Museo della Scuola e del libro per l'infanzia, Turin: Museo dell'Educazione, Padoue. Norvège (1): Bergen Skolemuseum,
Bergen. Pays-Bas (1): Nationaal Onderwijsmuseum, Rotterdam. Portugal (1): Museu Escolar dos Marrazes, Leiría. République tchèque (1): Pedagogické muzeum J.A. Komenského v Praze, Prague. Serbie (1): Pedagoški muzej, Belgrade. Slovaquie (1): Múzeum školstva a pedagogiky (MŠP), Bratislava. Slovénie (1): Slovenski Šolski Muzej, Ljubljana. Suisse (1): Schulmuseum Mühlebach, Amriswill. 
Il convient toutefois de reconnaître que notre corpus est loin d'être homogène et que certains musées ne remplissent pas tous les critères.

\section{Un champ muséal difficile à cerner}

La visibilité des musées de l'école et de l'éducation est encore mal assurée et leur caractère muséal n'est pas reconnu internationalement. Ces musées ne forment pas de comité international au sein de l'ICOM alors qu'il existe un comité international pour les musées et les collections universitaires (UMAC). Cet état de fait est la conséquence de l'absence de réelle définition, reconnue à l'échelle internationale, de ce qu'est un musée de l'école et de l'éducation et de l'absence de consensus des professionnels et des spécialistes en sciences de l'éducation sur ce qu'est ou doit être un "vrai » musée de l'école`. Le seul point de convergence, bien ténu, est qu'un tel musée doit conserver les traces matérielles sans lesquelles toute histoire de l'école est impossible et les interroger dans une perspective historique.

Le champ muséal reste mal identifié. Une des preuves de cette labilité est la difficulté à dresser une typologie des musées de l'école et de l'éducation actuels qui parvienne à englober tous les types de musées par-delà leur diversité de statuts, de missions, de moyens, de publics et de présentation. Juilo Ruiz Berrio, chercheur au Département de théorie et d'histoire de l'éducation et directeur du Museo de Historia de la Educación Manuel Bartolomé Cossío de l'Université Complutense de Madrid, en propose une qui trahit le regard d'un chercheur soucieux de déterminer le meilleur type d'institution pour fournir des sources au service de la recherche ${ }^{10}$. Il distingue cinq types de musées de l'école et de l'éducation: le "musée salle de classe", centré sur une salle de classe conservée in situ avec son mobilier et son matériel; le "musée d'histoire de l'enseignement", installé dans un bâtiment sans passé scolaire qui donne à voir les grandes étapes de l'histoire de l'enseignement ; le "musée de l'éducation", caractérisé par une exposition organisée autour d'un récit historique problématisé, proposant des reconstitutions de salles de classe,
9

Des essais de définitions théoriques ont pourtant été entrepris et portaient notamment sur les musées de l'école allemands, les Schulmuseen.

(AMLUNG, Ullrich, Jürgen

HELMCHEN et Uwe SANDFUCHS. Das Schulmuseum. Aufgaben, Konzeptionen und Perspektiven [Le musée de l'école. Mission, conception et perspective]. Weinheim und München: Juventa Verlag, 1997.) L'objet du doctorat d'Ulla M. Nitsch était de définir le Schulmuseum. L'auteure propose une modélisation théorique qui s'aligne très largement sur les définitions internationales proposées par l'ICOM. (NITSCH, Ulla M. Schule wandert ins Museum. Eine kritische Rekonstruktion der Musealisierung von Schul- und Pädagogikgeschichte 1977-1997 [L'école va au musée. Reconstruction critique de la muséalisation de l'histoire de l'école et de la pédagogie 1977-1977]. Berlin: Weidler Buchverlag, 2001.) 10

BERRIO, Julio Ruiz. "Pasado, presente i porvenir de los museos de educación " [Passé, présent et devenir des musées de l'éducation]. La memoria y el deseo. Cultura de la escuela y educación deseada [La mémoire et le désir. L'univers culturel et les projets éducatifs de l'école]. Valencia: Tirant lo Blanch, 2002, p. 43-65 
s'intéressant aux méthodes pédagogiques utilisées et présentant des matériels didactiques tout en fournissant des éclairages sur l'éducation actuelle. Le quatrième modèle est celui des "musées pédagogiques rénovés", qui mettent à la fois leurs ressources à la disposition des chercheurs en histoire ou en sciences de l'éducation et des enseignants en formation ou en activité et qui proposent $\mathrm{au}$ "grand public" une exposition sur l'histoire de l'enseignement. Enfin, le dernier type identifié est celui des "musées laboratoires de l'histoire de l'éducation", qui à la fois conservent des collections pouvant servir de sources pour la recherche, mènent des recherches en histoire de l'éducation et organisent des expositions temporaires dont les thématiques sont directement inspirées des problématiques de la recherche universitaire. Ce modèle représente pour l'auteur l'idéal du musée de l'éducation du XXIe siècle, même s'il souligne qu'il nécessite espace, personnel et budget conséquents. Cette grille de lecture certes séduisante s'avère, dans les faits, difficilement applicable (même si l'on s'en tient aux 29 musées européens étudiés) et n'épuise pas la gamme des possibles.

Malgré leurs diversités réelles qui rendent difficile toute étude comparative et leur manque de visibilité, des tentatives de structuration des musées de l'école et de l'éducation ont été entreprises depuis une trentaine d'années. Elles doivent beaucoup aux rencontres entre responsables de musées et aux relations interpersonnelles qui se sont développées à partir des années 1980 dans un contexte marqué par une éclosion de musées de l'école en Europe. En 1984, l'Internationales Symposium für Leiter und Betreuer von Schulmuseen und Schulgeschichtliche Sammlungen, à l'initiative de Rudolph Lukschanderl, fondateur du musée de l'école de Michelstetten en Autriche, réunit pour la première fois des équipes de musées de l'école. Jusqu'à la fin des années 1990, les participants sont majoritairement germanophones. Rebaptisés en allemand "Internationales Symposium für Schulmuseen und Schulhistorische (puis Schulgeschichtliche) Sammlungen " et en anglais "International Symposium for School Museums and School History Collections", ces rassemblements deviennent 
progressivement des forums d'échanges et de réflexions sur la nature, l'histoire, les missions, les pratiques et les activités des musées de l'école et des collections de patrimoine scolaire". Ils s'ouvrent à l'international et se professionnalisent. Une volonté de reconnaissance et de pérennisation aboutit en 2009 à la création d'un groupement européen d'intérêt économique, "Preservation of the School History Heritage in Europe EEIC / Bewahrung der materiellen Schulkultur in Europa EWIV", dont l'objectif est de promouvoir les musées de l'école et de l'éducation grâce à l'organisation de rencontres internationales, d'expositions et de bases de données en ligne.

Au-delà des diversités, il existe néanmoins une dimension commune partagée par ces institutions. Toutes affirment, en effet, un lien avec le patrimoine éducatif ou scolaire et visent à sa conservation, sa mise en valeur et sa présentation au public. Elles affirment implicitement que l'école et l'éducation sont dignes d'entrer dans un musée et que les objets qui en témoignent ont un caractère patrimonial. La valeur du patrimoine scolaire et du patrimoine éducatif reste assurément en deçà de celle reconnue aux domaines artistiques, historiques ou religieux. La question de l'identification et de la reconnaissance du patrimoine scolaire se pose d'ailleurs dans de nombreux pays ${ }^{12}$. Selon Anik Meunier, "au Québec, encore en ce jour, le patrimoine scolaire n'a pas franchi l'étape de reconnaissance. Les biens culturels qui le constituent ne sont pas identifiés ni reconnus comme ayant une valeur patrimoniale. "Celle-ci ajoute que "l'attention portée au patrimoine scolaire n'est pas non plus encore passée de la sphère privée (personnes, communautés, municipalités) à la sphère collective " ${ }^{13}$. Ce dernier point semble valoir pour l'Europe où le patrimoine scolaire, objet des soins de nombreux passionnés, ne bénéficie actuellement de l'intérêt que d'une minorité de collectivités territoriales et de peu de gouvernements régionaux ou nationaux. Or, sans cette reconnaissance publique, il ne peut y avoir de sauvegarde ni de transmission. Les musées de l'école et de l'éducation ne peuvent pas œuvrer seuls, sans le soutien de la puissance publique, pour légitimer la valeur du patrimoine scolaire et favoriser sa reconnaissance.
11

Ulla M. NITSCH y a présenté les thèmes abordés dans son travail de doctorat (op. cit., p. 43-85). 12

Cette question était au cœur du colloque Le patrimoine scolaire, un patrimoine à conserver? qui s'est tenu à l'Université du Québec à Chicoutimi en mai 2005. L'interrogation était suscitée par le constat, maintes fois réitéré, que le patrimoine scolaire souffrait d'un manque d'identification et de légitimité qui menaçait sa sauvegarde. Ce colloque a été organisé conjointement par le Centre interuniversitaire d'études québécoises (CIEQ) de l'Université Laval, dirigé par Brigitte Caulier, et le Groupe de recherche sur l'éducation et les musées (GREM) de l'Université du Québec à Montréal (UQAM), dirigé par Michel Allard. 13

MEUNIER, Anik. «Préface: Le patrimoine scolaire entre rupture et continuité". Le patrimoine scolaire: sa sauvegarde et sa valorisation. Montréal: Éditions MultiMondes, 2006, p. XVII. 
La reconnaissance sociale du patrimoine scolaire restant fragile et parfois inexistante, ce dernier reste de ce fait menacé.

\section{Les musées de l'école et de l'éducation en Europe: collections et fonctions}

Les musées de l'école et de l'éducation sont les héritiers directs, indirects ou spirituels des musées pédagogiques et scolaires nés au XIX $X^{e}$ siècle pour accompagner la mise en place des systèmes scolaires et la scolarisation obligatoire. Dans plusieurs pays d'Europe, ces musées figurent parmi les plus anciennes institutions muséales, bien qu'à l'origine la fonction conservatoire soit secondaire et s'efface devant la volonté de contribuer à l'amélioration de l'enseignement par la diffusion de matériels novateurs et de ressources auprès de professionnels de l'enseignement. Les musées pédagogiques et scolaires constituent un des modèles des musées du XIX ${ }^{\text {e }}$ siècle qui se diffuse jusque dans les années 1920 en Europe et dans les pays industrialisés, avant de connaître un déclin une fois les systèmes scolaires installés, déclin accéléré par les deux guerres mondiales qui souvent ont anéanti définitivement des structures aux moyens modestes et aux soutiens limités et ont éparpillé ou détruit les collections accumulées.

Les années 1980 voient l'éclosion d'une nouvelle génération de musées, portant parfois le même nom générique de "musées de l'école", dans un contexte radicalement différent, marqué par le bouleversement et la remise en question du système scolaire hérité du XIX siècle devenu "traditionnel". Ces musées d'un genre nouveau ne revendiquent pas de filiation avec les musées pédagogiques ni d'ailleurs ne la rejettent. Leur objectif est en tout cas opposé à celui de leurs devanciers tout comme l'est le regard porté sur les humbles objets scolaires. Il s'agit alors de sauver, pour les conserver et les faire connaître, les traces (notamment matérielles) et les bâtiments d'un monde scolaire en train de disparaître et qui se chargent progressivement d'une valeur patrimoniale et identitaire. Cet élan de création se poursuit pendant les deux décennies 
suivantes, porté par l'engouement croissant pour le patrimoine, pressé par la nécessité de trouver un avenir à des collections, parfois quantitativement importantes, rassemblées par des particuliers ou par des associations, et de les pérenniser. Ce mouvement est soutenu par l'intérêt croissant de chercheurs et d'universitaires spécialistes en histoire de l'éducation (et secondairement en sciences de l'éducation) qui considèrent désormais les collections de patrimoine scolaire et éducatif comme des sources irremplaçables pour la recherche.

$\mathrm{Au}$ terme d'une histoire longue de plus d'un siècle, qui connut ses périodes d'essor (entre 1850 et 1914 puis dans les années 1980), mais aussi de repli, voire de déclin (notamment entre 1930 et 1970), les musées de l'école et de l'éducation ont accumulé des collections quantitativement importantes et d'une diversité réelle, qui témoignent de l'histoire de l'enseignement plus que de celle de l'éducation. L'école primaire des années 1880-1950 y occupe la place la plus évidente, preuve de l'importance symbolique accordée à cette institution qui fut l'acteur majeur de la scolarisation obligatoire et de la formation d'une conscience nationale. Divers ensembles documentaires sont conservés - imprimés, archives, travaux d'élèves manuscrits, matériels didactiques tridimensionnels, documents sonores, audiovisuels et filmiques, matériels informatiques, mobiliers, vêtements -, même si quantitativement les imprimés l'emportent sur les objets tridimensionnels dans la plupart des collections. Les campagnes de collectes actives menées notamment dans les années 1970 et plus ponctuellement à l'occasion de nouvelles créations, tout comme les appels aux dons et les acquisitions onéreuses, ont permis de sauver de la disparition les humbles objets scolaires. Ces vastes entreprises semblent connaître un temps d'arrêt à la fin des années 2000. Les politiques d'enrichissement sont plus souvent passives qu'actives et prospectives car le souci actuel est avant tout de maîtriser au mieux les collections déjà constituées. 
majeure partie des institutions. Les outils de gestion permettant de maîtriser intellectuellement et physiquement les collections sont ainsi à construire ou à compléter. Ce travail colossal, notamment pour des structures qui fonctionnent avec des équipes réduites, nécessitera encore quelques décennies avant d'aboutir à un catalogage informatisé complet ainsi qu'à des bases partagées et mises en ligne. A l'heure actuelle, l'incapacité des institutions étudiées à savoir précisément ce qu'elles conservent est généralisée et les exceptions rares. Dans le domaine de la conservation également des améliorations sont à apporter pour atteindre les standards muséaux admis et connus des responsables de collections, qui, sensibilisés à ces problématiques, n'ont pas encore élaboré de politiques de conservation précises, inscrites dans la durée, le plus souvent faute de disposer d'une vision d'ensemble sur les fonds. De grandes disparités existent en matière de gestion de collections, tant en ce qui concerne les locaux, les équipements et les matériaux de conservation que les pratiques en matière de conservation préventive et de restauration. Les engagements financiers très conséquents dont a bénéficié le Musée national de l'éducation pour son chantier des collections (2008-2010) et la construction de nouveaux locaux abritant les collections, les activités d'accueil des chercheurs et les bureaux font exception.

Les musées de l'école et de l'éducation étudiés dans notre recherche doctorale ont dépassé le modèle des musées pédagogiques et scolaires qui, bien qu'ouverts au public, s'adressaient prioritairement à des spécialistes de l'enseignement à qui ils offraient des ressources. Ils proposent les formes traditionnelles de valorisation des collections que sont les expositions, les actions culturelles et les publications. Leur singularité réside dans les relations croissantes avec la recherche et dans la nature des publications où dominent quantitativement les productions destinées aux spécialistes. L'importance des animations destinées aux scolaires reposant pour beaucoup sur des leçons historiques et des jeux de rôle constitue une autre spécificité qui rappelle que le public majoritaire de ces musées est le public scolaire. 
Pour les musées de notre corpus, la seule fonction muséale partagée, outre la conservation, est celle d'exposer des collections. Les deux autres fonctions principales, à savoir la fonction scientifique et la fonction d'animation, peuvent ne pas être exercées ${ }^{14}$. Les expositions permanentes et temporaires proposées visent prioritairement à transmettre des connaissances sur l'histoire de l'école, de l'enseignement et, plus rarement, sur celle de l'éducation. L'approche historique, tournée vers le passé, l'emporte et imprime sa marque sur la scénographie, qui est dans l'ensemble très classique et qui parfois transforme l'exposition en un livre d'histoire illustré. L'analyse selon des critères muséographiques met en évidence la faiblesse de l'interactivité en décalage complet avec les attentes du jeune public majoritaire, tout comme l'hésitation entre des scénographies minimalistes et des scénographies réellement à décor. Les éléments scénographiques relevant de la scénographie à décor se limitent le plus souvent à l'incontournable salle de classe historique reconstituée et évoquée, simplement donnée à voir sans discours. D'autres lacunes peuvent être identifiées, tant dans la construction du scénario que dans la faible variété des supports d'information largement dominés par l'écrit et de qualité variable. Dans ce domaine aussi, l'hétérogénéité l'emporte. Néanmoins, sur le plan des contenus, des convergences s'observent. Dans l'ensemble, même s'ils sont riches et scientifiquement fondés, les discours sont factuels et peu problématisés. Surtout, ils restent bien souvent étrangers à l'actualité et aux questions de société.

L'analyse des publics montre la faible audience des musées de l'école ou de l'éducation. Près de 30 ans après leur création, les "nouveaux" musées de l'école et de l'éducation ne sont pas parvenus à diversifier leurs publics et connaissent des fréquentations modestes. Les uns se sont spécialisés dans l'accueil des scolaires auxquels ils consacrent l'essentiel de leur programmation culturelle. Certains autres, appartenant à des instituts de recherche, ont tenté de concilier, dans leurs expositions et leurs activités, les attentes d'un grand public et d'un public de spécialistes sans réellement y parvenir. Leur notoriété tant à l'échelle
14

Nous avons, par souci de clarté et en nous appuyant sur la définition du musée de l'ICOM, retenu quatre grandes fonctions muséales : conservation, exposition, recherche et animation. 
locale et régionale que nationale est très limitée. L'image dont ils jouissent reste attachée à celle de la salle de classe historique et à celle du musée poussiéreux traitant d'un sujet, l'école, qui n'évoque pas nécessairement de bons souvenirs à chacun. Depuis les années 2000, un nouveau modèle de musée de l'école et de l'éducation, centré réellement sur les publics, est néanmoins en train de se dessiner. Il se développe avec succès au Nationaal Onderwijsmusum de Rotterdam. L'enjeu est de taille pour les petites structures aux moyens limités que cette étude a fait le choix volontaire de ne pas traiter. La génération des fondateurs des années 1980 qui ont porté leur projet avec passion est sur le point de disparaître sans que, très souvent, ne soit assuré de relais. Plus généralement, la vague de patrimonialisation qui a porté les créations de musées de l'école et de l'éducation dans les années 1980-1990 est très certainement un phénomène historiquement circonscrit.

\section{Quel avenir pour les musées de l'éducation?}

C'est à dessein que nous avons choisi d'utiliser le terme de "musée de l'éducation" que nous distinguons de "musée de l'école». L'éducation devrait être réellement au cœur des thématiques du musée et les publics au cœur de ses missions. L'appellation musée de l'école est assurément trop réductrice et ne correspond que partiellement au contenu des expositions et des animations proposées. Elle véhicule des images teintées de nostalgie qu'il conviendrait de dépoussiérer et a l'inconvénient d'associer deux univers qui peuvent être répulsifs : celui des musées et celui de l'école. De nouveaux noms sont à trouver.

Les musées de l'éducation s'ils veulent rester vivants doivent continuer à enrichir leurs collections en menant de réelles politiques cohérentes qui prennent en compte l'éducation dans toutes ses dimensions, scolaire et extrascolaire, ses traces matérielles et immatérielles, son passé et son présent. Les collections actuelles des musées de l'école et de l'éducation du corpus font encore peu de place au patrimoine immatériel et ne documentent guère les années postérieures à 1960, années qui correspondent à la 
massification de l'enseignement. Documenter l'état actuel de l'éducation nécessite d'élaborer une méthodologie de collecte et de s'appuyer sur des partenaires. L'impossibilité d'embrasser l'ensemble de ce que recouvre l'éducation est évidente et oblige à faire des choix et des sélections explicitées et raisonnées. La conservation partagée, telle qu'elle a été mise en place depuis 2004 par l'Association des musées et collections historiques sur l'éducation en Suisse, est certainement une voie à développer car elle permet aux institutions partenaires de se spécialiser chacune dans certains domaines thématiques, chronologiques et géographiques. Cette spécialisation demandée par la législation muséale en Norvège et aux Pays-Bas a également l'avantage d'offrir une identité plus affirmée à chaque institution qui peut ainsi plus légitimement concentrer ses efforts et ses moyens financiers sur certaines collections-phares qui sont privilégiées en termes de traitement documentaire, de numérisation, de conservation, de restauration et d'acquisition. La mutualisation des moyens semble également s'imposer en matière de conservation et de restauration en raison des coûts, mais aussi de la spécialisation requise.

La dimension muséale doit être réaffirmée sans pour autant rompre avec la recherche. Cette dernière doit, en effet, venir appuyer, guider et étayer les choix en matière de politique d'enrichissement et de valorisation. L'alignement sur les standards muséaux et le respect de la législation patrimoniale permettraient aux institutions de gagner en clarté et d'être mieux identifiés par les partenaires et par les publics.

Il est également urgent et nécessaire de placer les publics au cœur des musées. C'est le positionnement adopté par les grandes institutions muséales héritées du siècle passé qui se sont restructurées récemment en France ou qui ont choisi d'affirmer cette politique. Être réellement ouverts à tous les publics suppose, pour les musées de l'école et de l'éducation actuels, de parvenir à attirer d'autres publics que les scolaires. Connaître les publics actuels et leurs attentes, mais aussi identifier de nouveaux publics cibles et leurs attentes s'impose comme des préalables 
nécessaires. Il convient également d'élaborer une offre adaptée aux publics cibles et diversifiée en liant expositions, animations, activités culturelles et publications. Du fait de leurs thématiques, les musées de l'éducation ont certainement un rôle plus spécifique à jouer vis-à-vis du jeune public (enfants, adolescents, scolaires), des parents, des enseignants en formation et en activité et des éducateurs. Les musées doivent s'ouvrir sur la société et prendre en compte ses attentes et ses interrogations sans pour autant céder aux effets de mode et aux discours dominants concernant l'éducation. Ils doivent au contraire mettre en perspective les débats actuels en les insérant dans le temps long et en nourrissant les réflexions par un retour sur le passé, appuyé sur des objets et sur l'histoire de l'éducation. Ils pourraient ainsi servir de référence en offrant un discours distancié et scientifiquement fondé. Opter pour le temps long permettrait également de démythifier la période 1880-1930 et de rendre intelligible le présent qui est souvent absent des expositions permanentes.

Les musées de l'éducation doivent aussi être des lieux vivants et évolutifs et retrouver ainsi l'esprit des fondateurs des musées pédagogiques qui souhaitaient en faire des espaces d'expérimentation en prise avec le présent. Cela passe par une attention accrue portée au confort de la visite et aux services proposés aux visiteurs. Cela suppose également des programmations culturelles plus diversifiées, qui sont adaptées à différents types de publics et dans lesquelles les débats et les échanges ont toute leur place. Cela nécessite enfin des présentations qui impliquent davantage le visiteur et qui l'amènent à se questionner, à prendre position et à exercer son esprit critique. Les dispositifs interactifs doivent être adaptés au public, tenir compte de son âge et de son bagage culturel. Des scénographies soignées qui osent les décors, surprennent, interrogent et misent sur les sens et la diversité des supports d'information seraient à privilégier. Des témoins vivants ou des témoignages oraux et filmés, plus efficaces que les mannequins costumés pour évoquer le passé, pourraient être utilisés plus systématiquement. La présentation et surtout l'utilisation de la salle de classe historique devraient être 
repensées. Appartenant désormais au passé, la salle de classe historique doit être accompagnée d'un discours qui permette de faire comprendre une forme historique d'enseignement spatialement délimitée. Des comparaisons systématiques entre des salles de classe de différentes époques reconstituées selon les mêmes critères scientifiques, comme à la Michelstettner Schule ou au Nationaal Onderwijsmuseum, seraient particulièrement éclairantes. On pourrait également imaginer, ce que ne fait aucun musée du corpus, des comparaisons géographiques et dépasser ainsi la dimension nationale, encore très largement dominante même dans les expositions temporaires qui se prêtent pourtant mieux aux comparaisons.

Exposer l'éducation, c'est exposer un acte de transmission qui ne se déroule pas nécessairement dans le cadre scolaire et qui mobilise un savoir, un savoir-faire ou un savoir-être, un éducateur et un apprenant. Ces deux acteurs doivent être représentés, alors que les expositions ont tendance à s'intéresser prioritairement au savoir et secondairement aux élèves.

L'exemple du Nationaal Onderwijsmuseum montre qu'une démarche centrée sur les publics est possible et que des bénéfices tangibles en matière de fréquentation et de notoriété peuvent être espérés assez rapidement. Cela nécessite certes des moyens matériels et financiers, des compétences humaines et des appuis institutionnels (qui actuellement font défaut), mais aussi et surtout de l'audace pour diversifier les sources de financement, se faire connaître et reconnaître. La mise en réseaux des musées de l'éducation et leur structuration permettraient assurément de renforcer chaque institution et de lui donner davantage de visibilité. L'enjeu actuel est réel: il en va de la pérennité des institutions au moment où la génération des fondateurs des musées de l'école des années 1980 se retire (souvent sans que la relève ne soit assurée) et où le champ culturel devient un secteur économique à part entière.

Le modèle des musées de l'école défini dans les années 1980, caractérisé par la primauté accordée à la sauvegarde 
du patrimoine scolaire, l'engagement individuel et la faiblesse des moyens, est aujourd'hui dépassé. Un nouveau modèle est à inventer. Au terme de notre étude, il nous semble que le musée de l'éducation de la décennie à venir doit s'affirmer comme un musée scientifique, inséré dans des réseaux, thématique et tourné vers les publics. Scientifique, il entretient des relations privilégiées avec les chercheurs et les spécialistes de l'éducation afin de se positionner comme un lieu de référence en matière de conservation et de connaissance du patrimoine éducatif. Inséré dans des réseaux et ouvert sur l'international, il noue des partenariats pour mener des recherches, des expositions, des actions de conservation, de restauration et d'enrichissement partagé des collections. Thématique, il traite de l'éducation au sens large, comprenant non seulement l'enseignement mais aussi la formation dite informelle dispensée et reçue dans l'univers extrascolaire et notamment familial. Tourné vers les publics, il propose une politique de valorisation ambitieuse, en prise avec les débats citoyens. Renouant avec les missions fondatrices des musées pédagogiques, il participe également à la formation initiale et continue des enseignants et des professionnels de l'enseignement dans un contexte où cette formation connaît de profondes transformations. Son projet scientifique et culturel doit être articulé autour des collections et des publics. Il fixe les missions suivantes : connaître les collections par un catalogage exhaustif accompagné de récolements ; les conserver dans des conditions optimales; les enrichir par une politique pluriannuelle cohérente qui repose sur des collectes thématiques et ciblées et ne néglige pas la mémoire orale; les étudier et les mettre à la disposition des chercheurs; les diffuser auprès de publics divers (experts, grand public, scolaires et étudiants, enseignants et spécialistes de l'éducation); les présenter grâce à des expositions adaptées aux publics visés qui, par leur caractère scientifique et sans céder au sensationnalisme, proposent des éclairages aux débats actuels; les valoriser par des animations pédagogiques et culturelles adaptées aux publics. 
Les musées de l'école et de l'éducation:

un champ muséal quantitativement

significatif mais difficile à cerner 
Summary

Translated by Allana Carlyle

School and Education Museums:

A quantitatively significant museological field but difficult to define 
More than a century after the creation of the first pedagogic and scholastic museums, school and education museums have formed a distinctive field of museology since the 1980's. A synthesis of both analytical and comparative approaches has therefore become possible.

This paper will present an overview of the primary results of a doctoral dissertation defended in September 2009. The goal of the research was to study the collections and museography of a corpus of 29 school and education museums located in twenty different European countries selected based on their cultural and scientific reputations. Based on field work carried out between 2005 and December 2007, a standardized questionnaire and documentary resources, the research was conceived as a contribution to the study of the permanency and improved recognition of the collections. The research first presents a quantitative and qualitative overview of education heritage preserved on a European scale. It reviews the management of education heritage, its policies on growth and documentation as well as its promotion. The research attempts to demonstrate that museum collections and museography reveal both the goals of museal institutions as well as the state of society along with society's choices and questions.

European school and education museums today face a double challenge. The first is to ensure the permanency of their collections and the recognition of their social and heritage roles. In fact, their fragility was recently demonstrated with the fate of two museums of ancient corpus (both were heirs of pedagogic museums founded in the late nineteenth century), with significant collections and enjoying a certain reputation. While the Dansk Skolemusem Copenhague has been closed since August $1^{\text {st }}, 2008$, the National Museum of Education in Rouen has changed management and despite moving to new functional premises in September 2010, still remains without a true scientific and cultural project. The second challenge is to attract and diversify their general public. The search must continue for a new model for school and education museums that meets the expectations of both the general public and researchers. 